\title{
Determination and Correlation Analysis of Moral Choice Influencing Factors Among Ant Tribe Young Intellectuals Based on Cognitive Neuroscience*
}

\author{
Bo Liu ${ }^{1}$ \\ Southwest Petroleum University \\ Lichuan $\mathrm{Luo}^{2}$ \\ Southwest Petroleum University \\ Qianyin $\mathrm{Xiao}^{3}$ \\ Southwest Petroleum University
}

\begin{abstract}
This paper aims to identify the psychological features of ant tribe young intellectuals (ATYIs) and the influencing factors of the ATYIs' moral choice. Based on the Lawrence Kohlberg's stages of moral development, the author discussed the influencing factors of the ATYIs' moral choice from the perspective of cognitive neuroscience. Then, the psychological features and moral judgement abilities of the ATYIs were determined through field trips, interviews and a questionnaire survey on 904 college graduates across China. The questionnaire involves four psychological test scales, namely Positive PsyCap Questionnaire (PPQ), Symptom Checklist 90 (SCL-90), Adult Dispositional Hope Scale (ADHS), and Perceived Stress Scale (PSS). The research results show that the ATYIs' moral choice is influenced by psychological factors, and that, with the improvement of the psychological quality of the ATYIs, their moral judgement ability will be greatly enhanced, leading to better moral choices.
\end{abstract}

\section{Keywords}

Moral choice • Cognitive Neuroscience • Ant Tribe Young Intellectuals (ATYIs) • Psychological Factors

\footnotetext{
* The work was supported by the special fund for humanities and social sciences of southwest petroleum university (project no: 2017RW011).

${ }^{1}$ Correspondence to: School of Economics and Management, Southwest Petroleum University, Chengdu 610500, China. Email: th100th@swpu.edu.cn

${ }^{2}$ School of Economics and Management, Southwest Petroleum University, Chengdu 610500, China. Email: 26285728@qq.com

${ }^{3}$ Foreign Language School, Southwest Petroleum University, Chengdu 610500, China. Email: conniexqy@qq.com

Citation: Liu, B., Luo, L. C., \& Xiao, Q. Q, Determination and Correlation Analysis of Moral Choice Influencing Factors Among Ant Tribe Young Intellectuals Based on Cognitive Neuroscience. Educational Sciences: Theory \& Practice, 18(5), 1483-1493. http://dx.doi.org/10.12738/estp.2018.5.045
} 
Recent years has witnessed a continuous growth in the number of college graduates in China. Since 2011, this number has increased year by year from $2 \%$ to $5 \%$. In 2017, the number of Chinese college graduates reached 7.95 million, an increase of 160,000 compared with the previous year. In the past 7 years, the number of college graduates in China has accumulated to a staggering amount of 50.75 million (www.chinabaogao.com). After entering the workforce, many college graduates become the so-called "ant tribe young intellectuals" (ATYIs), i.e. "a group of low-income university graduates". They are viewed as the main force of social construction.

The term ATYIs is a derivation from the buzzword "ant tribe". The latter first appeared in the book Ant Tribe authored by Lian Si, referring to low-paid new college graduates aged between 22 and 30 and living in rented houses within urban-rural junctions or urban villages. However, it is improper to describe this specific group of people with such a general phrase. After all, common urban residents and migrant workers also fit in with the common definition of ant tribe: low-income youngsters living in shabby rented houses. Hence, the college graduates should beredefined as the ATYIs, forming the fourth most vulnerable group in China after peasants, migrant workers and laid-off workers (Baidu Encyclopaedia). The moral development of the ATYIs is an important part of the moral education of Chinese youth. After all, the moral choice can determine the individual behaviours, which directly bear on the occurrence and development of social behaviours (Arbit, Ruby $\&$ Sproesser, 2017). To enhance the moral education in colleges, it is necessary to ascertain the influencing factors and evolution law of the moral choice of the ATYIs (Berwick, 2017).

Morality has long been a hot topic among scholars at home and abroad. For example, Lawrence Kohlberg explored the moral level of individuals through moral dilemmas (Baidu Encyclopaedia). However, the exploration stops at the external moral judgement, rather than consider whether the individual moral choice is affected by psychological factors (Boccia et al., 2017). In fact, the mental state is a reaction of brain cells to external stimuli. The brain cells are capable of making scientific and rational response to various external stimuli. The reaction is a silence cognitive process, which externalizes itself as individual behaviors (Liu, Luo \& Xiao, 2018). Studies have shown that, once the cognitive mapping is established in the brain, cognitive activities will activate he brain cells, induce the brain potential, and stimulate the conduction of brain nerves, so that the brain will allocate resources to create emotions and brain images (Journal of Cognitive Neuroscience). In return, the above process will influence the individual behaviors. By this theory, the moral choices of the ATYIs, as the external behaviors, must be impacted by the internal psychological cognition (Chamik, Viswanathan, Gedeon, \& Bovet 2017).

Foreign scholars have probed deep into moral decision-making. As early as 2004, Joshan Greene and his colleagues suggested that people make different choices in moral dilemmas because emotional decision varies with the degrees of personal involvement. Greene et al. compared those of low personal involvement and those of high personal involvement in moral dilemmas through functional magnetic resonance imaging (FMRI). As expected, the personal and non-personal decisions are associated with the activation of different brain regions. In general, non-personal decisions are made when the right lateral prefrontal cortex and bilateral parietal lobe (the brain regions of working memory) are activated. By contrast, personal decisions are made when the medial frontal cortex, posterior cingulate gyrus, and amygdala are obviously activated. To sum up, the moral decision 
is closely correlated with our emotional cognition of what is morally acceptable (Xiao, 2018). Since emotion is a manifestation of mental state, the moral decisions of the ATYIs must have something to do with their mental state (Yoo, Sanders \& Cerveny, 2018). The subject's response of moral judgement can be determined by the moral judgment ability test (MJAT) (Nickelsen, 2018).

In light of the above, this paper aims to answer the following questions: (1) What are the psychological features of the ATYIs. (2) Whether the moral choice of the ATYIs is influenced by psychological factors? (3) If yes, what are the effects of psychological factors on the moral choice of the ATYIs? Considering China's national conditions and social development, four moral dilemmas were created to test the moral judgement ability of the ATYIs. Meanwhile, four psychological test scales were adopted to evaluate the mental state of the ATYIs, including the Positive PsyCap Questionnaire (PPQ), Symptom Checklist 90 (SCL-90), Adult Dispositional Hope Scale (ADHS), and Perceived Stress Scale (PSS). The research framework is shown in Figure 1.

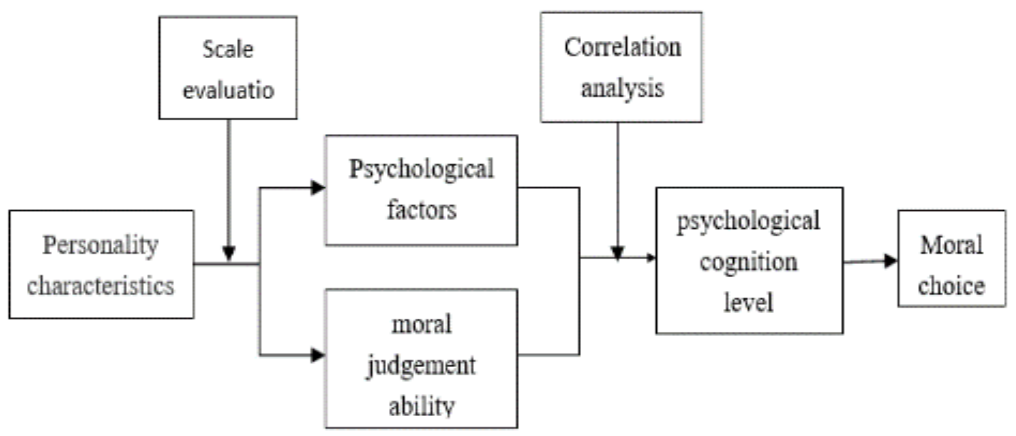

Figure 1. Research framework.

\section{Methodology}

\section{Sampling}

The ATYIs were surveyed through field visits, interviews and an online questionnaire survey. To eliminate interference of human factors and protect the privacy of the respondents, a total of 1,013 questionnaires was prepared on wjx.com, and issued online to those graduated from colleges across China in the past five years. In the end, $904(89.2 \%)$ valid questionnaires were returned. The questionnaire mainly asks about the mental state and moral choice of the respondents. The samples were mostly collected from provincial capitals and other large cities, which are the strongholds of new college graduates.

\section{Variable Measurement}

Four widely accepted psychological test scales were employed to evaluate the mental state of the respondents. Besides, a highly credible and effective MJAT scale was adopted from the articles published on A-level journals in China. 


\section{MJAT scale}

The MJAT scale mainly tests the moral judgement ability of the respondents. The scale was prepared based on the "Preparation and Norm Formulation of Moral Judgement Ability Test for Shanghai Youth. Considering China's national conditions and social development, four moral dilemmas were created as follows (Salloch, 2016),Below is a summary of the four moral dilemmas.: (1) Dilemma 1: To repay an obligation, a centre forward decides to throw the game so that the opponent coach won't be sacked; (2) Dilemma 2: A husband is forced to steal medicine to save the life of his dying wife; (3) Dilemma 3: An ailing old expert chooses conservative treatment so that his impoverished students could learn more knowledge and make a better living; (4) Dilemma 4: A young girl insists on spending a sum of money she saved to buy a show ticket rather than pay tuition (as her mother wishes). For each dilemma, approval is rated as 1 and disapproval as 2 . The rating is positively correlated with the individual moral judgement ability. Here, the MJAT has a reliability (0.905) and a high validity (0.961), indicating that the scale is suitable for factor analysis.

\section{PPQ}

The PPQ is a 26-item test scale that measures the positiveness and optimism (e.g. "I have better than average insights and abilities", and "I can recover quickly from setbacks."). This scale contains four factors, namely, hope, optimism, self-efficacy and resilience (Stoner, Orrell \& Spector, 2018). The items are rated against a 7 point Likert scale (1=strongly disagree; $7=$ strongly agree). Here, the PPQ has a reliability $(0.927)$ and a high validity $(0.910)$, indicating that the scale is suitable for factor analysis.

\section{SCL-90}

The SCL-90 is one of the world's most famous mental health test scales. It is currently the most popular mental disorder and mental illness checklist (Eich et al., 2012). This scale involves 90 items in the following 10 dimensions: somatization, obsessive-compulsive, interpersonal sensitivity, depression, anxiety, hostility, phobic anxiety, paranoid ideation, psychoticism, and others (Sogou Encyclopaedia). It weighs the mental symptoms of the respondents from ten aspects (e.g. headaches and nervousness). The items are rated between 1 (no symptom) and 5 (severe symptom). Here, the SCL-90 has a reliability (0.983) and a high validity (0.956), indicating that the scale is suitable for factor analysis.

\section{ADHS}

The ADHS is a 12-item scale that measures the individual expectations (e.g. "I am tirelessly pursuing my goals.”). The scale consists of two parts: will power and pathway thinking (Keefer \& Laurie, 2018). The items are rated between 1 (absolutely incorrect) to 4 (absolutely correct). Here, the ADHS has a reliability (0.878) and a high validity (0.786), indicating that the scale is suitable for factor analysis. 


\section{PSS}

The PSS is a 13-item scale that measures the respondents' ability to withstand stress (e.g. "How often do you feel that you are losing control of the important things in life?"). The scale has three stress scenarios: daily trivia, major events and resource-induced changes (Klein et al., 2016). The items are rated between 1 (often) to 5 (never). The score is positively correlated with the stress-bearing ability. Here, the PSS has a reliability (0.945) and a high validity (0.902), indicating that the scale is suitable for factor analysis.

\section{Statistical processing}

The SPSS 24.0 was adopted for data analysis, including single-sample t-test and correlation analysis.

\section{Data Analysis and Results}

\section{Single-sample t-test}

The four scales were respectively subjected to single-sample t-test, aiming to disclose the psychological features of the ATYIs. The test results include the mean value (M), standard deviation (SD), t-value and significance (p-value), and 95\% confidence interval of the difference. These indices can fully demonstrate the data features and verify whether there is a significant difference between the sample mean and the population mean). The single-sample t-test results of the PPQ, SCL-90, ADHS and PSS are respectively listed in Tables $1 \sim 4$.

As shown in Table 1, the four dimensions of the PPQ were significantly different at the level of 0.01 $(\mathrm{P}<0.01)$. The mean scores of the ATYIs were 4.65, 4.53, 5.07 and 5.24, respectively, for optimism, resilience, hope, and self-efficacy. The low scores for optimism and resilience manifest that the ATYIs are not good at coping with setbacks, and prone to mental imbalance and low confidence facing the changes in the external environment (Van, Morse \& Flory, 2015). The high scores for hope and self-efficacy indicate that the ATYIs are hopeful about the future, confident in their abilities, and convicted that they can realize their dreams with their efforts and abilities (Honey, Lynch, Burke, \& Gilmour, 2011).

As shown in Table 2, the 10 factors of the SCL-90 were significantly different at the level of $0.01(\mathrm{P}<0.01)$. Among them, the scores for somatization (1.31) and terror (1.32) were significantly lower than the normal levels. This is because the ATYIs, aged between 22 and 30, are generally in good health and are less fearful in the face of dangers (Rosenberg et al., 2018) [16]. On the contrary, the scores for obsessive-compulsive symptom (1.80), interpersonal sensitivity (1.58), and depression (1.52) were significantly higher than the normal levels. Hence, the ATYIs are under more stress than the average person, and more susceptible to influence when handling interpersonal relationships, which results in a strong sense of discomfort. Compared with ordinary people, the ATYIs are highly likely to be depressed facing the high pressure and sensitive interpersonal relationships (Wang, 2018). 
As shown in Table 3, the four dimensions of the ADHS were significantly different at the level of 0.01 $(\mathrm{P}<0.01)$. The mean scores for pathway thinking (2.97) and will power (2.76) were both significantly higher than the normal levels, suggesting that the ATYIs have richer goals and more diverse ways to achieve them than the ordinary people (Lewis, 2017).

Table 1

Single-Sample t-Test Results of the PPQ

\begin{tabular}{lcccccc}
\hline \multirow{2}{*}{ Projects } & \multirow{2}{*}{$\mathrm{M}$} & \multirow{2}{*}{$\mathrm{SD}$} & \multirow{2}{*}{ T value } & Significant (P value) & \multicolumn{2}{c}{ 95\% confidence interval of difference } \\
\cline { 6 - 7 } Optimism & 4.65 & 0.96 & 146.06 & 0.00 & 4.59 & 4.71 \\
Resilience & 4.53 & 0.87 & 156.42 & 0.00 & 4.48 & 4.59 \\
Hope & 5.07 & 1.04 & 145.96 & 0.00 & 5.00 & 5.14 \\
Self-efficacy & 5.24 & 0.96 & 164.28 & 0.00 & 5.18 & 5.31 \\
\hline
\end{tabular}

Table 2

Single-Sample t-Test Results of the PPQ

\begin{tabular}{lcccccc}
\hline \multirow{2}{*}{ Projects } & $\mathrm{M}$ & $\mathrm{SD}$ & $\mathrm{T}$ value & $\begin{array}{c}\text { Significant } \\
\text { (P value) }\end{array}$ & \multicolumn{2}{c}{ 95\% confidence interval of difference } \\
\cline { 6 - 7 } Somatization & 1.31 & 0.49 & 79.39 & 0.00 & 1.27 & Lower limit \\
Obsessive-compulsive & 1.80 & 0.63 & 86.33 & 0.00 & 1.76 & 1.34 \\
Interpersonal sensitivity & 1.58 & 0.62 & 76.74 & 0.00 & 1.54 & 1.84 \\
Depression & 1.52 & 0.61 & 74.60 & 0.00 & 1.48 & 1.56 \\
Anxiety & 1.43 & 0.55 & 78.28 & 0.00 & 1.40 & 1.47 \\
Hostility & 1.46 & 0.58 & 75.27 & 0.00 & 1.42 & 1.50 \\
Phobic anxiety & 1.32 & 0.52 & 75.93 & 0.00 & 1.28 & 1.35 \\
Paranoid ideation & 1.40 & 0.53 & 79.79 & 0.00 & 1.37 & 1.44 \\
Psychoticism & 1.41 & 0.53 & 80.54 & 0.00 & 1.37 & 1.44 \\
Others & 1.44 & 0.53 & 82.00 & 0.00 & 1.41 & 1.47 \\
\hline
\end{tabular}

Table 3

Single-Sample t-Test Results of the ADHS

\begin{tabular}{lcccccc}
\hline \multirow{2}{*}{ Projects } & M & SD & T value & $\begin{array}{c}\text { Significant } \\
\text { (P value) }\end{array}$ & 95\% confidence interval of difference \\
\hline Pathway thinking & 2.97 & 0.41 & 216.81 & 0.00 & Lower limit & Upper limit \\
Will power & 2.76 & 0.50 & 165.53 & 0.00 & 2.94 & 2.99 \\
\hline
\end{tabular}

Table 4

Single-Sample t-Test Results of the PSS

\begin{tabular}{lcccccc}
\hline \multirow{2}{*}{ Projects } & M & SD & $\begin{array}{c}\text { T } \\
\text { value }\end{array}$ & $\begin{array}{c}\text { Significant } \\
\text { (P value) }\end{array}$ & \multicolumn{2}{c}{$\begin{array}{c}\text { 95\% confidence interval of } \\
\text { difference }\end{array}$} \\
\cline { 6 - 7 } & & & & & Lower limit & Upper limit \\
\hline Daily trivia & 3.24 & 0.71 & 137.64 & 0.00 & 3.19 & 3.28 \\
Major events & 3.35 & 0.71 & 142.61 & 0.00 & 3.31 & 3.40 \\
Resource-induced changes & 3.15 & 0.73 & 129.91 & 0.00 & 3.10 & 3.19 \\
\hline
\end{tabular}

As shown in Table 4, the three dimensions of the ADHS were significantly different at the level of 0.01 $(\mathrm{P}<0.01)$. The mean scores for daily trivia, major events and resource-induced changes were respectively 3.24 , 3.35 and 3.15. The high score for major events reveals that the ATYIs can identify the major events under pressure out of the maze of events in life, and handle them well (Chamik, Viswanathan, Gedeon, \& Bovet 2017), while the low score for resource-induced changes indicate that the lifestyle, physical state and mental state of the ATYIs are vulnerable to environmental changes, i.e. the ATYIs are sensitive internally (Wang, Dong and Dai, 2011). 


\section{Correlation analysis}

The correlation analysis was conducted separately against the MJAT, PPQ, SCL-90, ADHS and PSS to judge whether the moral choice of the ATYIs is influenced by their psychological factors and what are the exact impacts. The results are included in Tables 5 8, respectively. Note that $\mathrm{P}<0.01$ means the correlation is significant at the level of 0.01 , while $\mathrm{P}<0.05$ means the correlation is statistically significant at the level of 0.05 .

In Dilemma 1, the moral choice of the subjects had a significant positive correlation with the PPQ mean score $(r=0.07, p<0.05)$, the ADHS mean score $(r=0.08, p<0.05)$ and the PPS mean score $(r=0.10, p<0.01)$, and a significant negative correlation with the SCL-90 mean score $(r=-0.13, \mathrm{p}<0.01)$.

In Dilemma 2, the moral choice of the subjects had a significant positive correlation with the PPQ mean score $(r=0.10, p<0.01)$ and the ADHS mean score $(r=0.11, p<0.01)$, a significant negative correlation with the SCL-90 mean score $(r=-0.13, p<0.01)$, and no significant correlation with the PSS mean score.

In Dilemma 3, the moral choice of the subjects had a significant positive correlation with the PPQ mean score $(r=0.10, p<0.01)$, a significant negative correlation with the SCL-90 mean score $(r=-0.13, p<0.01)$, and no significant correlation with the ADHS mean score or the PSS mean score.

In Dilemma 4, the moral choice of the subjects had a significant positive correlation with the PPQ mean score $(r=0.07, p<0.05)$, a significant negative correlation with the PSS mean score $(r=0.08, p<0.05)$, and no significant correlation with the SCL-90 mean score or ADHS mean score.

Table 5

Correlations between Moral Choice and Psychological Factors for Dilemma 1

\begin{tabular}{lccccccr}
\hline Variable & $\mathrm{M}$ & $\mathrm{SD}$ & 1 & 2 & 3 & 4 & 5 \\
\hline 1.PPQ & 4.87 & 0.81 & 1 & & & & \\
2.SCL-90 & 1.47 & 0.51 & $-0.41^{* *}$ & 1 & & & \\
3.ADHS & 2.80 & 0.41 & $0.60^{* *}$ & $-0.46^{* *}$ & & & \\
4.PSS & 3.27 & 0.66 & $0.48^{* *}$ & $-0.59^{* *}$ & $0.64^{* *}$ & 1 & \\
5.Dilemma 1 moral choice & 1.53 & 0.50 & $0.07^{*}$ & $-0.13^{* *}$ & $0.08^{*}$ & $0.10^{* *}$ & 1 \\
\hline
\end{tabular}

Table 6

Correlations between Moral Choice and Psychological Factors for Dilemma 2

\begin{tabular}{lccccccc}
\hline Variable & $\mathrm{M}$ & $\mathrm{SD}$ & 1 & 2 & 3 & 4 & 5 \\
\hline 1.PPQ & 4.87 & 0.81 & 1 & & & & \\
2.SCL-90 & 1.47 & 0.51 & $-0.41^{* *}$ & 1 & & & \\
3.ADHS & 2.80 & 0.41 & $0.60^{* *}$ & $-0.46^{* *}$ & & & \\
4.PSS & 3.27 & 0.66 & $0.48^{* *}$ & $-0.59^{* *}$ & $0.64^{* *}$ & 1 & \\
5.Dilemma 2 moral choice & 1.62 & 0.49 & $0.10^{* *}$ & $-0.13^{* *}$ & $0.11^{* *}$ & 0.07 & 1 \\
\hline
\end{tabular}

Table 7

Correlations between Moral Choice and Psychological Factors for Dilemma 3

\begin{tabular}{lccccccc}
\hline Variable & $\mathrm{M}$ & $\mathrm{SD}$ & 1 & 2 & 3 & 4 \\
\hline 1.PPQ & 4.87 & 0.81 & 1 & & & & \\
2.SCL-90 & 1.47 & 0.51 & $-0.41^{* *}$ & 1 & & & \\
3.ADHS & 2.80 & 0.41 & $0.60^{* *}$ & $-0.46^{* *}$ & & \\
4.PSS & 3.27 & 0.66 & $0.48^{* *}$ & $-0.59^{* *}$ & $0.64^{* *}$ & 1 \\
5.Dilemma 3 moral choice & 1.81 & 0.39 & $0.12^{* *}$ & $-0.12^{* *}$ & -0.00 & 0.03 & 1 \\
\hline
\end{tabular}


Table 8

Correlations between Moral Choice and Psychological Factors for Dilemma 4

\begin{tabular}{lccccccc}
\hline Variable & $\mathrm{M}$ & $\mathrm{SD}$ & 1 & 2 & 3 & 4 & 5 \\
\hline 1.PPQ & 4.87 & 0.81 & 1 & & & & \\
2.SCL-90 & 1.47 & 0.51 & $-0.41^{* *}$ & 1 & & & \\
3.ADHS & 2.80 & 0.41 & $0.60^{* *}$ & $-0.46^{* *}$ & & & \\
4.PSS & 3.27 & 0.66 & $0.48^{* *}$ & $-0.59^{* *}$ & $0.64^{* *}$ & 1 \\
5.Dilemma 4 moral choice & 1.37 & 0.48 & $0.07^{*}$ & -0.04 & 0.04 & $0.07 *$ & 1 \\
\hline
\end{tabular}

Next, the correlations between moral choice and psychological factors for the four dilemmas were summarized (Table 9). As shown in Table 9, the moral choice of the respondents had a significant correlation with the scores of all four scales for Dilemma 1, the scores of PPQ, SCL-90 and ADHS for Dilemma 2, the scores of PPQ and SCL-90 for Dilemma 3, and the scores of PPQ and PSS for Dilemma 4.

Table 9

Correlations between moral choice and psychological factors for all four dilemmas

\begin{tabular}{lcccc}
\hline Projects & PPQ & SCL-90 & ADHS & PSS \\
\hline Dilemma 1 moral choice & $0.07^{*}$ & $-0.13^{* *}$ & $0.08^{*}$ & $0.10^{* *}$ \\
Dilemma 2 moral choice & $0.10^{* *}$ & $-0.13^{* *}$ & $0.11^{* *}$ & 0.07 \\
Dilemma 3 moral choice & $0.12^{* *}$ & $-0.12^{* *}$ & -0.00 & 0.03 \\
Dilemma 4 moral choice & $0.07^{*}$ & -0.04 & 0.04 & $0.08^{*}$ \\
\hline
\end{tabular}

\section{Discussion}

\section{Research conclusions}

The four scales in this research reflect the overall mental state of the ATYIs. Through the single-sample ttests against the four scales, it is possible to draw the psychological features of the ATYIs: they have full confident of their abilities and adopt various means to achieve their goals; however, they are not good at withstanding stress and are easily depressed due to the discomfort resulted from external influences. Thus, the first question raised in our research is answered.

According to the correlation analysis against the MJAT and the four psychological scales, the moral choice of the ATYIs in the four moral dilemmas has a significant correlation with the mean scores of the four scales, indicating that the ATYIs' moral choice is affected by various psychological factors. Thus, the second question raised in our research can be answered: the ATYIs' moral choice is influenced by psychological factors (Guo, Gu \& Su, 2017).

To sum up, the respondents' moral choice in dilemmas is clearly positively correlated with optimism, hope and stress-bearing ability, and negatively with psychological symptoms. Hence, it can be concluded that: with the improvement of the psychological quality of the ATYIs (i.e. the increase in optimism, hope and stressbearing ability and decrease in psychological symptoms), their moral judgement ability will be greatly enhanced, leading to better moral choices. Thus, the third question raised in our research is answered. 


\section{Theoretical significance}

Although Lawrence Kohlberg has also discussed the moral development of individuals in moral dilemmas, his discussion stops at individual level (Bordignon, 2011). This paper proves that the moral judgement ability can be studied from the perspective of psychological cognition, in addition to the individual level (Peens \& Louw, 2000). In our research, the moral choice and psychological factors of the ATYIs were subjected to interactive analysis, revealing that the individual moral choice is affected by psychological factors.

\section{Practical significance}

Our research discovers that the ATYIs' moral choice is affected by psychological factors, and their moral judgement ability increases with the improvement of psychological quality. Therefore, the moral education in Chinese colleges should attach equal importance to the cultivation of behaviours and mental health. Moreover, our discussion on the psychological features of the ATYIs can provide a reference for psychological education in colleges (Biebel et al., 2018).

\section{Research limitations}

There are still some limitations in this research: (1) The samples were collected from some, not most of the large cities in China. The limited scope may harm the credibility of our samples. The future research will expand the scale and scope of sampling. (2) The scales in our research are mostly based on Western theories, which may not fully apply to the situation in China.

\section{References}

Arbit, N, Ruby, M. B., \& Sproesser, G. (2017). Spheres of moral concern, moral engagement, and food choice in the USA and Germany. Food Quality \& Preference, 62. 38-45, http://dx.doi.org/ 10.1016/j.foodqual.2017.06.018.

Berwick, D. M. (2017). Moral choices for today's physician. JAMA, 318(21), 2081-2082, http://dx.doi.org/ 10.1001/jama.2017.16254

Biebel, K., Ryder-Burge, A., Alikhan, S., Ringeisen, H., \& Ellison, M. (2018). Strategies to Support the Education Goals of Youth and Young Adults with Serious Mental Health Conditions: A Case Study. Administration and Policy in Mental Health and Mental Health Services Research, 45(4), 661671. http://dx.doi.org/ 10.1007/s10488-018-0852-3.

Boccia, M., Verde, P., Angelino, G., Carrozzo, P., Vecchi, D., Piccardi, L., ... \& Giannini, A. M. (2017). Effect of professional expertise and exposure to everyday life decision-making on moral choices. Neuroscience letters, 654, 80-85. http://dx.doi.org/ 10.1016/j.neulet.2017.06.036. 
Chamik, T., Viswanathan, B., Gedeon, J., \& Bovet, P. (2018). Associations between psychological stress and smoking, drinking, obesity, and high blood pressure in an upper middle-income country in the African region. Stress and Health, 34(1), 93-101. http://dx.doi.org/ 10.1002/smi.2766.

Eich, D., Angst, J., Frei, A., Ajdacic-Gross, V., Rössler, W., \& Gamma, A. (2012). A new rating scale for adult ADHD based on the Symptom Checklist 90 (SCL-90-R). European Archives of Psychiatry and Clinical Neuroscience, 262(6), 519-528. http://dx.doi.org/ 10.1007/s00406-011-0288-1.

Guo, H., Gu, S., \& Su, Y. (2018). Bargaining power choices with moral hazard in a supply chain. Discrete Dynamics in Nature and Society, 2018, 2, 1-9. http://dx.doi.org/ 10.1155/2018/2068287.

Honey, J., Lynch, C. D., Burke, F. M., \& Gilmour, A. S. M. (2011). Ready for practice? A study of confidence levels of final year dental students at Cardiff University and University College Cork. European Journal of Dental Education, 15(2), 98-103. http://dx.doi.org/ 10.1111/j.16000579.2010.00646. x.

Keefer, L. (2018). Behavioural medicine and gastrointestinal disorders: the promise of positive psychology. Nature Reviews Gastroenterology \& Hepatology, 15, 378-386. http://dx.doi.org/ 10.1038/s41575-018-0001-1.

Klein, E. M., Brähler, E., Dreier, M., Reinecke, L., Müller, K. W., Schmutzer, G., ... \& Beutel, M. E. (2016). The German version of the Perceived Stress Scale-psychometric characteristics in a representative German community sample. BMC Psychiatry, 16(1), 159. http://dx.doi.org/ 10.1186/s12888-016-0875-9.

Lewis, E. F. (2017). The hope that early detection can tip the scale towards heart failure prevention. Jacc Heart Fail, 5(3), 191-93. http://dx.doi.org/ 10.1016/j.jchf.2017.02.001.

Liu, B., Luo, L., \& Xiao, Q. (2018). Determination and correlation analysis of mental health influencing factors among ant tribe young intellectuals based on cognitive neuroscience. NeuroQuantology, 16(5), 901-906, http://dx.doi.org/ 10.14704/nq.2018.16.5.1324.

Nickelsen, K. (2018). On Otto Warburg, Nazi Bureaucracy and the difficulties of moral judgment. Photosynthetica 56, 75-85. http://dx.doi.org/ 10.1007/s11099-018-0773-0.

Rosenberg, A. R., Bradford, M. C., Bona, K., Shaffer, M. L., Wolfe, J., Baker, K. S., ... \& Yi-Frazier, J. (2018). Hope, distress, and later quality of life among adolescent and young adults with cancer. Journal of Psychosocial Oncology,36(2), 137-144. http://dx.doi.org/ 10.1080/07347332.2017.1382646.

Salloch, S. (2016). Professionals watching TV and the question of moral supererogation. American Journal of Bioethics, 16(11), 54-56. http://dx.doi.org/ 10.1080/15265161.2016.1222013.

Stoner, C. R., Orrell, M., Spector, A. (2018). The positive psychology outcome measure (ppom) for people with dementia: Psychometric properties and factor structure. Archives of Gerontology \& Geriatrics, 76, 182-87. http://dx.doi.org/ 10.1016/j.archger.2018.03.001. 
Van, E. K., Morse, M., \& Flory, K. (2015). The role of body image in the link between ADHD and depression symptoms among college students. Journal of Attention Disorders, 155(12), 4843-55. http://dx.doi.org/ 10.1177/1087054715580845.

Wang, C., Dong, X. M., \& Dai, J. F. (2011). Analysis on the incidence and risk factors of campus violence among college students in Guangzhou]. Zhonghua yu fang yi xиe za zhi [Chinese Journal of Preventive Medicine, 45(9), 778-84. http://dx.doi.org/ 10.3760/cmaj. issn.0253-9624.2011.09.003.

Wang, P. (2018). Determination of countermeasures for depression through interviews on young depression patients in anthropology. NeuroQuantology, 16(5). 907-914. http://dx.doi.org/ 10.14704/nq.2018.16.5.1403.

Xiao, G. (2018). Psychological mechanism of adolescent internet addiction and brain functional imaging. NeuroQuantology, 16(5), 915-20. http://dx.doi.org/ 10.14704/nq.2018.16.5.1422.

Yoo, C. W., Sanders, G. L., \& Cerveny, R. P. (2018). Exploring the influence of flow and psychological ownership on security education, training and awareness effectiveness and security compliance. Decision Support Systems, 108, 107-18. http://dx.doi.org/ 10.1016/j.dss.2018.02.009. 